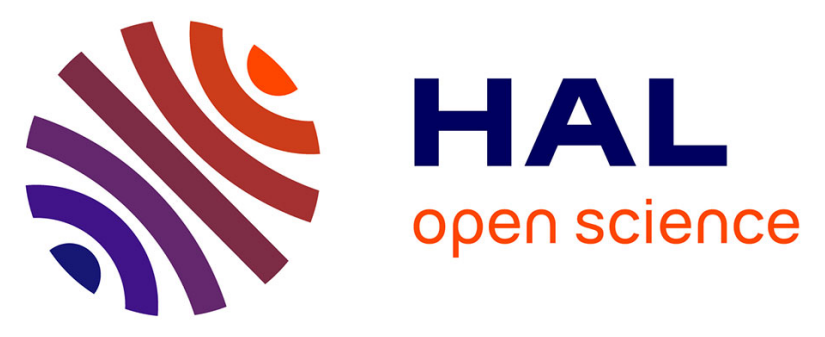

\title{
IUVS echelle-mode observations of interplanetary hydrogen: Standard for calibration and reference for cavity variations between Earth and Mars during MAVEN cruise
}

\author{
Majd Mayyasi, John Clarke, Eric Quémerais, Olga Katushkina, Dolon \\ Bhattacharyya, Jean-Yves Chaufray, Jean-Loup Bertaux, Bill Mcclintock, Ian \\ Stewart, Greg Holsclaw, et al.
}

\section{- To cite this version:}

Majd Mayyasi, John Clarke, Eric Quémerais, Olga Katushkina, Dolon Bhattacharyya, et al.. IUVS echelle-mode observations of interplanetary hydrogen: Standard for calibration and reference for cavity variations between Earth and Mars during MAVEN cruise. Journal of Geophysical Research Space Physics, 2017, 122 (2), pp.2089-2105. 10.1002/2016JA023466 . insu-01442123

HAL Id: insu-01442123

https://hal-insu.archives-ouvertes.fr/insu-01442123

Submitted on 8 Sep 2020

HAL is a multi-disciplinary open access archive for the deposit and dissemination of scientific research documents, whether they are published or not. The documents may come from teaching and research institutions in France or abroad, or from public or private research centers.
L'archive ouverte pluridisciplinaire HAL, est destinée au dépôt et à la diffusion de documents scientifiques de niveau recherche, publiés ou non, émanant des établissements d'enseignement et de recherche français ou étrangers, des laboratoires publics ou privés. 


\section{Journal of Geophysical Research: Space Physics}

\section{RESEARCH ARTICLE \\ 10.1002/2016JA023466 \\ Special Section: \\ Major Results From the MAVEN \\ Mission to Mars \\ IUVS echelle-mode observations of interplanetary hydrogen: Standard for calibration and reference for cavity variations between Earth and Mars during MAVEN cruise}

Key Points:

- IPH diffuse emission is used to

calibrate the MAVEN IUVS instrument

- Stable calibration factor is obtained to

facilitate interpretation of

observations

- Spectral characteristics of IPH observed by the MAVEN echelle instrument are consistent with previous observations

Correspondence to:

M. Mayyasi,

majdm@bu.edu

\section{Citation:}

Mayyasi, M., et al. (2017), IUVS echelle-mode observations of interplanetary hydrogen: Standard for calibration and reference for cavity variations between Earth and Mars during MAVEN cruise, J. Geophys. Res. Space Physics, 122, 2089-2105, doi:10.1002/2016JA023466.

Received 14 SEP 2016 Accepted 14 JAN 2017

Accepted article online 20 JAN 2017 Published online 2 FEB 2017

@2017. American Geophysical Union. All Rights Reserved.

\author{
Majd Mayyasi' ${ }^{1}$, John Clarke ${ }^{1}\left(\mathbb{D}\right.$, Eric Quémerais ${ }^{2}$, Olga Katushkina ${ }^{3}$ (D) Dolon Bhattacharyya ${ }^{1}$ (D), \\ Jean-Yves Chaufray ${ }^{2}$, Jean-Loup Bertaux ${ }^{2} \mathbb{D}$, Bill McClintock ${ }^{4}\left(\mathbb{D}\right.$, lan Stewart ${ }^{4}$, Greg Holsclaw ${ }^{4} \mathbb{D}$, \\ Justin Deighan ${ }^{4}$, Michael Chaffin ${ }^{4}$, Nick Schneider ${ }^{4}$ iD, and Bruce Jakosky ${ }^{4}$ iD \\ ${ }^{1}$ Center for Space Physics, Boston University, Boston, Massachusetts, USA, ${ }^{2}$ LATMOS/IPSL, Guyancourt, France, ${ }^{3}$ Space \\ Research Institute of Russian Academy of Sciences, Moscow, Russia, ${ }^{4}$ Laboratory for Atmospheric and Space Physics, \\ University of Colorado Boulder, Boulder, Colorado, USA
}

\begin{abstract}
The high-resolution echelle mode of the Imaging Ultraviolet Spectrograph (IUVS) instrument on the Mars Atmosphere and Volatile Evolution mission has been designed to measure D and H Lyman $\alpha$ emissions from the Martian atmosphere to obtain key information about the physical processes by which water escapes into space. Toward this goal, the absolute calibration of the instrument is critical for determining the $\mathrm{D}$ and $\mathrm{H}$ densities, the $\mathrm{D} / \mathrm{H}$ ratio, and the escape flux of water. The instrument made observations of interplanetary hydrogen (IPH) along multiple look directions and conducted several postlaunch calibration campaigns during cruise as well as during orbit around Mars. The calibration efforts monitored instrument degradation and produced a consistent calibration factor at the hydrogen Lyman $\alpha$ wavelength $(121.567 \mathrm{~nm})$. The instrument was calibrated with the diffuse emission of interplanetary hydrogen (IPH) as a standard candle using measurements and model results from the Solar Wind Anisotropies (SWAN) instrument. Validation of the calibrated instrument was made by (1) comparisons to simultaneous observations of the IPH made with the lower resolution FUV mode of the IUVS instrument that were independently calibrated by using standard stars and by (2) comparisons to same-day observations of Mars at hydrogen Lyman $\alpha$ made with the Hubble Space Telescope that were calculated with a radiative transfer model. Adopted FUV mode values and Hubble Space Telescope-based model results agreed with the echelle SWAN calibrated values to within $6 \%$ and $4 \%$, respectively. The calibrated IUVS instrument can be used to interpret emissions of atmospheric species at Mars for insights into water evolution at the planet, as well as observed IPH measurements made during cruise for further insights into dynamics of the inner heliosphere.
\end{abstract}

\section{Introduction}

The Mars Atmosphere and Volatile Evolution (MAVEN) mission's Imaging Ultraviolet Spectrograph (IUVS) is one of a suite of instruments designed to investigate the loss of atmospheric species at Mars [Jakosky, 2015]. IUVS is the first spacecraft-borne instrument to successfully utilize an ultraviolet echelle grating outside of Earth's orbit. The echelle grating enables the instrument to operate in high-resolution mode to spectrally resolve the emissions of hydrogen, at $121.567 \mathrm{~nm}$, and deuterium, at $121.534 \mathrm{~nm}$ [McClintock et al., 2014; Clarke et al., 2017]. This capability makes it possible to determine the D/H ratio in the upper Martian atmosphere, the region from which the escape of water constituents takes place. To be able to extrapolate back in time to relate the present elevated $\mathrm{HDO} / \mathrm{H}_{2} \mathrm{O}$ ratio in the lower atmosphere to the primordial water content on Mars, understanding of the physical processes by which water escapes today is needed [Villanueva et al., 2015]. To accomplish this, accurate calibration of the IUVS echelle instrument is essential to scientifically interpret its measurements.

In the far ultraviolet (FUV) wavelength range, the Martian atmosphere is observed as a mixture of optically thin and thick emissions. Optically thin (and typically weaker) emissions such as deuterium Lyman $\alpha$ follow the linear portion of the curve of growth. As a result, the observed absolute brightness of an optically thin line can be used to linearly interpret the line of sight (LOS) column density, and subsequently the local abundance, of that species in the atmosphere. Optically thick emissions such as those of hydrogen Lyman $\alpha$ and 


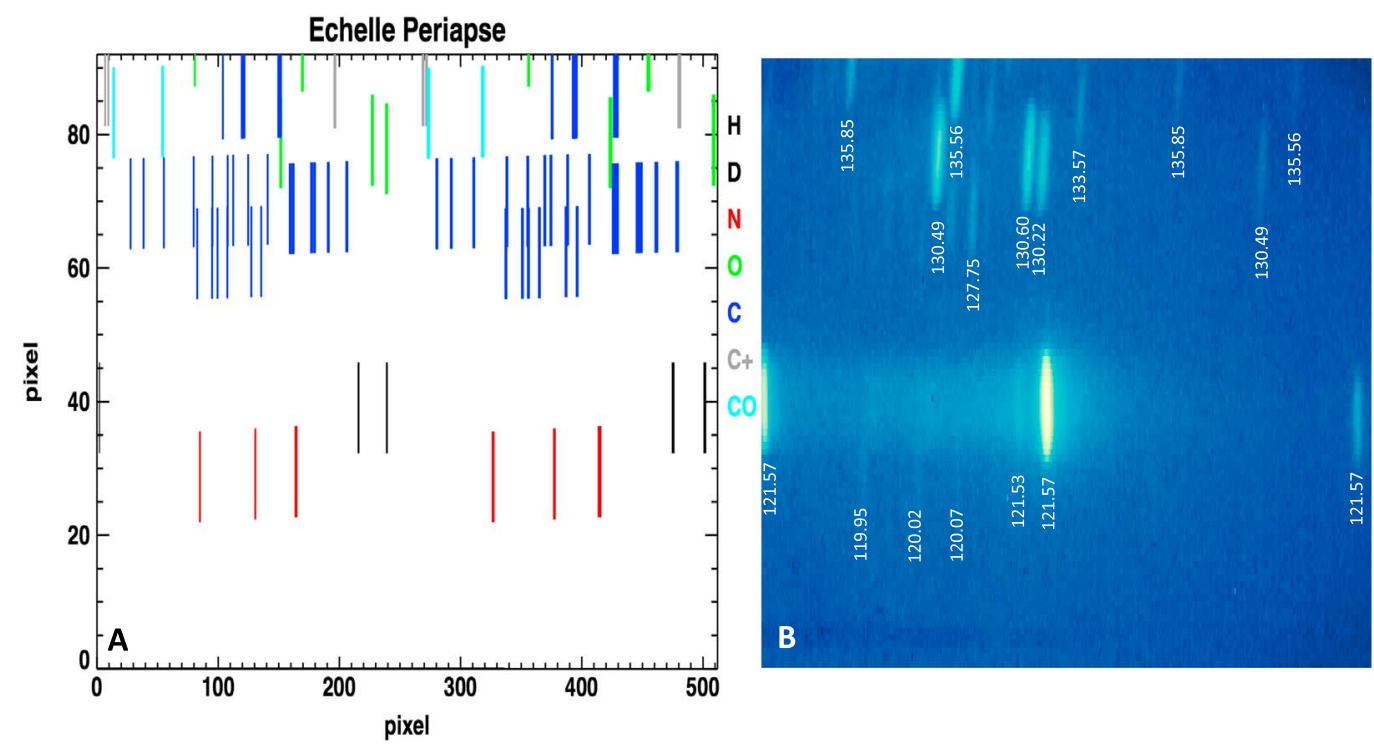

Figure 1. (a) $512 \times 92$ binned echellogram showing theoretical emission line locations as determined from instrument design, adjusted for in-flight observations. The colors of the lines represent the emitting species (black for $\mathrm{H}$ and $\mathrm{D}$ Lyman $\alpha$, red for the $\mathrm{N} 120 \mathrm{~nm}$ triplet, green for O 130.4 triplet and 135.6 doublet, blue for multiple $\mathrm{C} 127 \mathrm{~nm}$ emissions, grey for $\mathrm{C}^{+} 133 \mathrm{~nm}$ emission, and light blue for CO $131 \mathrm{~nm}$ emissions). The vertical lengths of the lines represent the projections of the aperture on the detector. The multiple horizontal aperture projections on the detector correspond to different orders of the same emission lines and are denoted as lines of different thicknesses. H Lyman $\alpha$, for example, appears in three orders: one at the center (order number 346), a second one to the far right, and a third to the extreme left edge of the image. (b) Echelle composite image (scaled logarithmically in intensity for clarity) of multiple periapse passes with a total integration time of $8.458 \mathrm{~h}$ completed across 5 weeks. Various emission lines are observed and labeled according to their wavelength (in nanometer). Specifically shown are emissions of hydrogen and deuterium Lyman $\alpha$ at 121.57 and $121.53 \mathrm{~nm}$, respectively; nitrogen at 119.95, 120.02, and $120.07 \mathrm{~nm}$; oxygen at 130.22, 130.49, 130.60, 135.56, and $132.85 \mathrm{~nm}$; and carbon $127.75 \mathrm{~nm}$ and singly ionized carbon at $133.57 \mathrm{~nm}$. Fainter line labels have been omitted for clarity.

several oxygen FUV lines are subject to self-absorption along the LOS, making the retrieval of abundances of these species more convoluted. Models are used to interpret the observed line of sight brightness of optically thick emissions to produce physical properties of atmospheric species such as number densities and temperatures. The accurate measurement of the absolute brightness of both optically thin and thick emission lines at Mars is therefore critical to understanding the abundance, variability, and evolutionary history of atmospheric species [Chaufray et al., 2009; Bhattacharyya et al., 2016]. The IUVS echelle instrument has been designed for this purpose.

The low-resolution modes of the IUVS instrument measure long aperture two-dimensional spectra of UV emissions, with wavelength in one dimension and spatial location in the other dimension. The IUVS highresolution echelle mode observations, shown in Figure 1, measure two-dimensional spectra, called echellograms, that project multiple spectral orders onto various regions on the detector separated both spatially and spectrally. The echelle optical layout is a custom design for this application, taking advantage of the fact that the Martian airglow spectrum at FUV wavelengths contains emission lines with no true (blackbody) continuum. In this case, the cross dispersion can be accomplished with a low-resolution prism, greatly simplifying the layout but requiring a limited aperture length to avoid order overlap of the main emission lines. The echelle grating in IUVS is a replica of the Hubble Space Telescope (HST) Space Telescope Imaging Spectrograph instrument E1 echelle, and the prism has been designed to allow wavelengths less than $140 \mathrm{~nm}$ onto the detector, avoiding contamination from longer wavelengths. The combination of aperture width and echelle dispersion results in a spectral resolution at Lyman $\alpha$ of $0.008 \mathrm{~nm}$ and a dispersion sampling of $0.00071 \mathrm{~nm} /$ pixel, producing a spectral resolution of $\sim 15000$ at $121.567 \mathrm{~nm}$. This is more than sufficient to separate the $\mathrm{H}$ and $\mathrm{D}$ Lyman $\alpha$ lines $(0.033 \mathrm{~nm}$ separation) as well as the multiplets of other emissions such as the OI 130.4 and $135.6 \mathrm{~nm}$ lines. The spectral sampling changes with binning scheme and is dictated by the bandwidth available for data download from the spacecraft. For echelle observations 


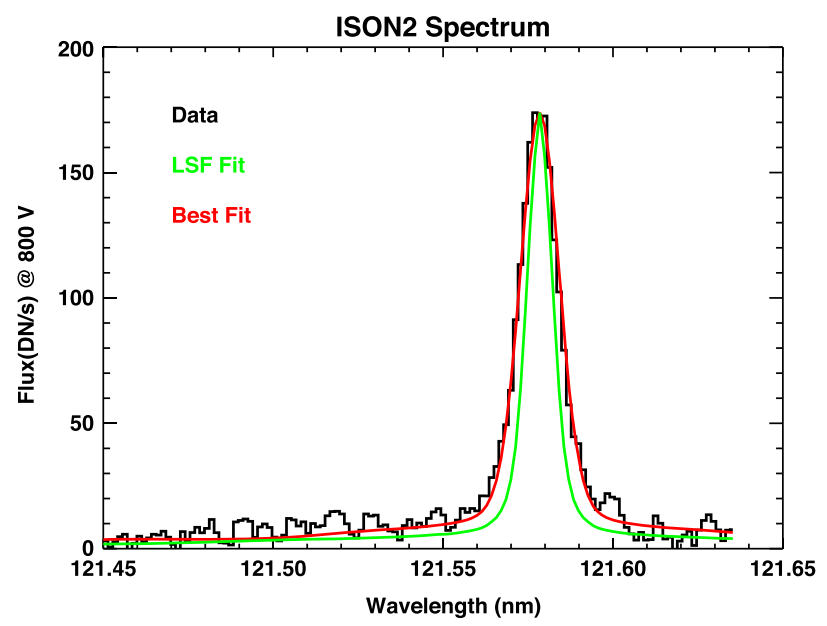

Figure 2. A sample IPH spectrum from the ISON2 epoch that was observed on 15 December 2013. For this epoch, five observations were made along similar lines of sight and using the same instrument constraints. Each observation included 36 integrations of $60 \mathrm{~s}$ duration. The black line is a coadded spectrum from the total $3 \mathrm{~h}$ of integration. The green line is the empirically determined instrument line spread function (LSF) that has a FWHM of $0.008 \mathrm{~nm}$ and has been scaled to the peak of the spectrum. The red line is the best fit curve to the data and is a result of convolving the instrument LSF with a Voigt profile to get a curve of FWHM of $0.015 \mathrm{~nm}$. detected count rate from $2 \times 11$ pixels on the full detector. Figure la shows a theoretical map of emission line projections on the detector determined by ray-tracing emission wavelengths through the optics. The aperture curvature is not included and affects only the shape of the line spread function that is intrinsic to the optical system, not the results of the calibration work described here. Figure $1 \mathrm{~b}$ is a compilation of approximately $8.5 \mathrm{~h}$ of integrated echelle observations made during the periapse segment of the spacecraft orbit around Mars. This figure shows the brightest emissions from the sunlit Martian upper atmosphere coming from the $\mathrm{H}$ Lyman $\alpha$ emission $(121.567 \mathrm{~nm})$ in the middle of the detector array and from the Ol triplet $(130.2,130.4$, and $130.6 \mathrm{~nm}$ ) plus the forbidden Ol doublet (135.6 and $135.8 \mathrm{~nm}$ ) emissions at the top of the detector array. In addition, Figure $1 \mathrm{~b}$ shows the grating scattered light extending horizontally from $\mathrm{H}$ and from the fainter $D$ Lyman $\alpha$ emission to the left of the $H$ line.

The echelle spectra are transformed into count rates in data numbers (DNs) per second as a function of wavelength in a way that is nuanced to the instrument settings. These corrections include spectral and spatial sampling of the detector, integration time, number of exposures, and voltage settings. Count rates from observed emission features are integrated across the aperture of the instrument as projected on the detector and converted into total flux. This conversion process is described further in the Appendix A. To summarize, the observed count rates are processed through a pipeline that removes background noise, scattered light, energetic particle effects, and faulty pixel effects from the images. The photons integrated across the aperture field of view and captured by the detector are summed up for each emission and then converted into standardized units of kilo-Rayleigh (kR) by using a conversion factor that encompasses the makeup and settings of the grating, and that remain fixed with time (instrument degradation notwithstanding). The calibrated fluxes of optically thin and thick emission lines, determined in kR, are then used with models to directly interpret the abundances and temperatures of atmospheric species at Mars [e.g., Bhattacharyya et al., 2015; Chaufray et al., 2015; Chaffin et al., 2015]. An example of the integrated count rate of the hydrogen Lyman $\alpha$ emission from observing the interplanetary medium is shown in Figure 2 and described in the next section.

The hydrogen corona at Mars extends to many Martian radii [Clarke et al., 2014]. The MAVEN spacecraft, during echelle mode operations, nominally orbits the planet at distances less than 1.5 Martian radii and therefore lies within the extended coronal exosphere of the planet. As a result, the Mars $\mathrm{H}$ emission line is observed by IUVS as spatially diffuse (aperture-filling) and optimally requires calibration with another 
diffuse source such as the interplanetary hydrogen emission (IPH). $\mathrm{H}$ Lyman $\alpha$ from interplanetary hydrogen is observed in most IUVS echelle spectra (with the exception being for observations of the Martian disk). IPH emissions can be spectrally resolved from the Martian $\mathrm{H}$ emission by the line of sight Doppler shift that is produced by the combination of IPH flow, LOS direction, and velocity of MAVEN.

The IPH Lyman $\alpha$ emission is produced by solar photons in the $121.567 \mathrm{~nm}$ resonance line scattered by interplanetary neutral hydrogen atoms. This ubiquitous emission varies with different factors such as solar activity, distance from the Sun, and the observer's line of sight [Quémerais et al., 2013, and references therein]. Neutral hydrogen from the interstellar medium flows through the heliosphere [e.g., Lallement et al., 2010]. This flow can Doppler shift the emission from the perspective of the observer by up to $0.017 \mathrm{~nm}$-compared to the full width at half maximum (FWHM) of the emission of $\sim 0.01 \mathrm{~nm}$ [Clarke et al., 1998]. Models of the IPH are therefore used to account for these and other variations in the direction, location, and velocity of the IPH flow along the observing instrument line of sight.

The Solar Wind Anisotropies (SWAN) instrument is one of the instruments on board the Solar and Heliospheric Observatory ( $\mathrm{SOHO}$ ) spacecraft that is dedicated to studying the IPH. As its name suggests, SWAN studies the anisotropies in the solar wind and IPH by making routine measurements of the whole sky [Bertaux et al., 1995]. These measurements are coupled with models to provide continuous estimates of IPH brightness throughout the solar system [Quémerais et al., 2008]. SWAN-based observations and models were used to cross calibrate the MAVEN IUVS echelle observations during cruise as well as after orbit insertion during five campaigns that targeted simultaneous measurements of diffuse emissions of hydrogen. SWAN itself was calibrated in absolute sensitivity, in part, by comparison with HST observations of the IPH close in time and angle on the sky [Quémerais et al., 2013]; thus, the SWAN calibration is derived from and consistent with that of HST.

The cross-calibration efforts described in this work are done for the Lyman $\alpha$ wavelength only $(121.567 \mathrm{~nm})$. Laboratory measurements of the IUVS instrument as well as low-resolution mode stellar calibration campaigns using stars as point-source standard candles indicated wavelength-dependent differences in instrument response between Lyman $\alpha$ and other detectable FUV wavelengths [McClintock et al., 2014]. This means that emission lines that fall on locations of the detector other than the central H Lyman $\alpha$ location will require additional corrections. These corrections are made to account for variations in instrument response that are sensitive to the echelle grating blaze function as well as to account for scattered light effects off the edges of the detector by flat-fielding the image. The echelle data reduction and calibration results, described in this work, take such adjustments into account for the central $\mathrm{H}$ and $\mathrm{D}$ Lyman $\alpha$ emissions.

The next section of this manuscript describes the calibration of the IUVS instrument. The resulting calibration was successfully validated by using two independent methods described in section 3. MAVEN IUVS echelle cruise observations were analyzed to provide calibrated values of the IPH thermal and flux distributions, as described in section 4. Results and discussion are presented in section 5. The details of echelle image processing and associated uncertainties are described in the Appendix A.

\section{Instrument Calibration}

The interplanetary medium emits an ultraviolet background at Lyman $\alpha$ due to resonant scattering of sunlight by neutral hydrogen atoms entering the solar system from the interstellar medium. This IPH Lyman $\alpha$ emission is used for cross calibration of the high-resolution echelle mode observations and the coarser resolution far ultraviolet channel (FUV-mode) observations of the IUVS instrument using SWAN observations.

\subsection{Echelle Calibration}

The far ultraviolet emissions of Mars' atmospheric species are observed as spatially diffuse. The IPH emissions were the brightest (and only diffuse) emissions observed by the IUVS echelle during the cruise phase of the MAVEN mission on the way to Mars. A spectrum of the IPH, measured by the IUVS echelle in December 2013, with its best fit curve are shown in Figure 2. Due to the scattered light in the instrument and thermal distribution of atoms in the IPH, the best fitting curve was found to be that of a combination of the instrument line spread function and a Voigt profile. Details of the image processing, fitting techniques, and observational details of the ISON2 data are described in more detail in the Appendix A. 


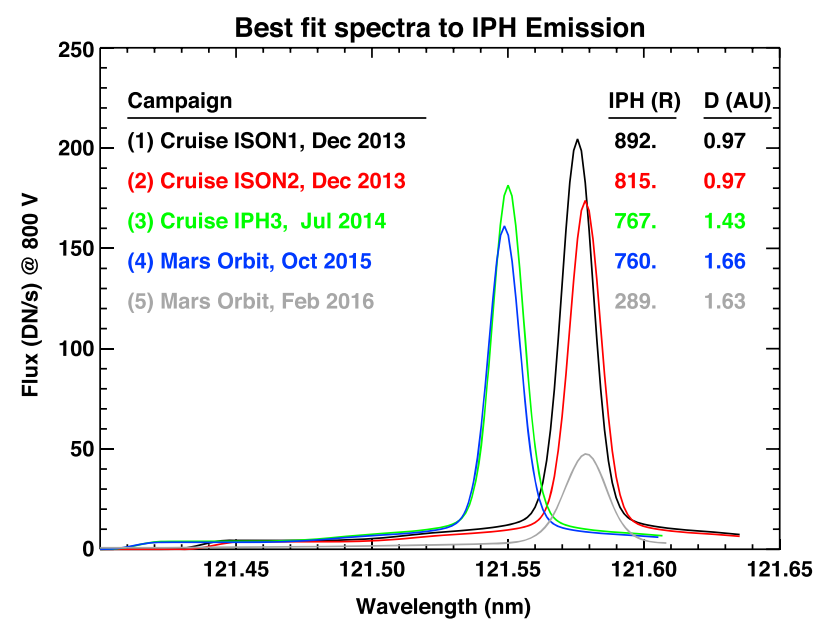

Figure 3. The best fit spectra to the IPH emission observed by MAVEN IUVS echelle. The observations have been normalized to the same detector gain and binning schemes for direct comparison. The legend on the left lists the observation epochs. The legend on the right lists the radiance of IPH as determined by SWAN, in Rayleighs, and that has been corrected for MAVEN's location and observation LOS. The spacecraft-Sun distance, in $\mathrm{AU}$, is also listed for each of the calibration campaigns. Variation in the location and flux of the peak of the IPH emission from each epoch are due to the Doppler shift of the IPH flow along the spacecraft line of sight velocity vector, as well as the variability of the IPH within the solar system. the flow mainly due to charge exchange with solar wind protons. To calculate the IPH distribution in the heliosphere, a three-dimensional time-dependent kinetic model developed by Izmodenov [2006] was used together with a radiative transfer model [Quémerais, 2000] to calculate the intensity of backscattered Lyman $\alpha$ radiation for different observer locations (SWAN and MAVEN) along their respective lines of sight to facilitate making corrections for SWAN-observed intensities.

Five campaigns were conducted to cross calibrate the echelle mode observations with SWAN results. In the MAVEN mission timeline, these campaigns were labeled according to their observation epochs as follows: (a) cruise phase ISON1, (b) cruise phase ISON2, (c) cruise phase IPH3, (d) Mars Orbit in October 2015, and (e) Mars Orbit in February 2016. The best fits to the IPH emission spectra observed by the echelle and the resulting radiances determined by SWAN for each of these five campaigns are shown in Figure 3.

The voltage settings across the detector varied across the five calibration campaigns. Additionally, the subset of the IUVS detector array that was downloaded, as well as the binning scheme, varied across the calibration campaigns. All observations were consistently processed through an image reduction pipeline, described in the Appendix A, to produce comparable results. Individual observations from each epoch were averaged to minimize noise levels and produce one spectrum. A fit was then made to the averaged spectrum. The resulting best fit spectra, shown in Figure 3, have been normalized to the same gain and scaled to similar binning schemes. The variations in the wavelength of the peak of the IPH emission are due to the Doppler shift of the interplanetary hydrogen with respect to the line of sight of the IUVS instrument. The variations in the flux of the peak of the spectra are due to the nonuniform IPH distribution throughout the interplanetary medium [Quémerais et al., 2006; Izmodenov et al., 2013].

The IPH spectra obtained during the calibration campaigns were fit to a curve produced by convolving a Voigt profile with the instrument line spread function (LSF). The width of the Voigt profile was varied (as well as its peak flux and location) for optimum fitting. The best fit curves to the spectra are shown in Figure 3. The best fit Voigt temperature (representative of the IPH line width), averaged over the epochspecific observations of each echelle calibration campaign, was found to be $17,500 \mathrm{~K}$ for the ISON $1 / 2$ epochs; $21,500 \mathrm{~K}$ for the IPH3 epoch; 17,000 K for the October 2015 epoch; and 31,000 K for the Feb 2016 epoch. These results represent apparent temperatures, since there is a spread in velocities of $\mathrm{H}$ atoms along the LOS. The 
Table 1. Results From Five IUVS Echelle and Two FUV Intercalibration Campaigns With SOHO-SWAN

\begin{tabular}{|c|c|c|c|c|c|c|c|c|c|c|c|}
\hline No. & Epoch & Date & $\begin{array}{l}\text { Gain (V) } \\
(D N / p h)^{a}\end{array}$ & $\begin{array}{c}\text { IPH Int. } \\
\text { Flux (DN/s) } \\
5 \times f w h m^{b}\end{array}$ & $\begin{array}{c}\text { IPH Int. } \\
\text { Flux }(\mathrm{Ph} / \mathrm{s}) \\
5 x f w h m^{\mathrm{b}}\end{array}$ & $\begin{array}{c}\text { SWAN } \\
\text { Brightness } \\
\text { (Rayleigh) }\end{array}$ & $\begin{array}{c}\text { SWAN } \\
\text { Correction } \\
\text { for MAVEN }\end{array}$ & $\begin{array}{l}\text { Corrected } \\
\text { Brightness } \\
\text { (Rayleigh) }\end{array}$ & $\begin{array}{c}\text { Cal } \\
\text { Factor } \\
(\mathrm{ph} / \mathrm{s} / \mathrm{kR})\end{array}$ & $\begin{array}{c}\text { Cal } \\
\text { Factor } \\
(\mathrm{DN} / \mathrm{s} / \mathrm{kR})\end{array}$ & $\begin{array}{l}\text { Cal Factor } \\
\text { (DN/s/kR) } \\
\text { (at } 800 \mathrm{~V})\end{array}$ \\
\hline \multicolumn{12}{|c|}{ Echelle } \\
\hline 1 & ISON1 & Dec 2013 & $850(248)$ & 7,350 & 29.6 & 892 & None & 892 & 33.2 & 8,237 & 3,948 \\
\hline 2 & ISON2 & Dec 2013 & $850(248)$ & 6,188 & 24.9 & 815 & None & 815 & 30.6 & 7,592 & 3,638 \\
\hline 3 & IPH3 & Jul 2014 & 800 (119) & 2,499 & 21.0 & 614 & $+25 \%$ & 767 & 27.4 & 3,258 & 3,258 \\
\hline 4 & Mars Orbit & Oct 2015 & 800 (119) & 2,513 & 21.1 & 886 & $-17 \%$ & 760 & 27.8 & 3,307 & 3,307 \\
\hline 5 & Mars Orbit & Feb 2016 & 800 (119) & 1,063 & 8.94 & 250 & $+19 \%$ & 289 & 30.0 & 3,564 & 3,564 \\
\hline \multicolumn{12}{|c|}{ FUV } \\
\hline 2 & ISON2 & Dec 2013 & 850 & 61,738 & 249 & 815 & None & 815 & 306 & 75,764 & 36,354 \\
\hline 3 & IPH3 & Jul 2014 & 800 & 18,079 & 152 & 614 & $+25 \%$ & 767 & 198 & 23,562 & 23,562 \\
\hline
\end{tabular}

${ }^{\mathrm{a}}$ Data numbers (DN) per photon (ph). Data number is the number of counts obtained from a level1a IUVS data product.

b Integrated IPH signal to within 5 FWHM of peak, and adjusted total to account for full signal, as described in the text.

CCorrected brightness refers to the SWAN-measured value at 1 AU that has been corrected by using a model to adjust for the location of the MAVEN spacecraft and the instrument LOS during the observation epoch.

range of best fit IPH temperatures is consistent with previous estimates of the IPH temperatures, measured from Earth's orbit [Clarke et al., 1998]. It is noted that the resulting IUVS-derived IPH temperatures are on the higher end of the range of expected values and this is discussed further in section 4.

A summary of the calibration properties determined from the echelle campaigns is listed in Table 1. A summary of operational parameters (such as voltage settings and binning schemes) and observational details (such as right ascension and declination along the measurement LOS) for the five echelle calibration campaigns are described in the Appendix A and summarized in Tables A1 and A2, respectively.

\subsection{FUV Calibration}

Two cross-calibration campaigns were conducted by using IUVS FUV-mode observations and SWAN as constrained by LOS with both echelle and SWAN observations. These occurred for in-cruise epochs (b) ISON2 and (c) IPH3 described in the echelle calibration section. During epoch (a) described in the previous section, the FUV pointing did not align with that of the echelle. During calibration campaigns (d) and (e), MAVEN was in orbit around Mars, and the FUV observations are not able to spectrally resolve the IPH from Mars $\mathrm{H}$ emissions.

The IPH emissions observed by the IUVS FUV mode for campaigns (b) and (c) are shown in Figure 4 for comparison. The spectra have been normalized to the same detector gain and binning scheme for comparison.

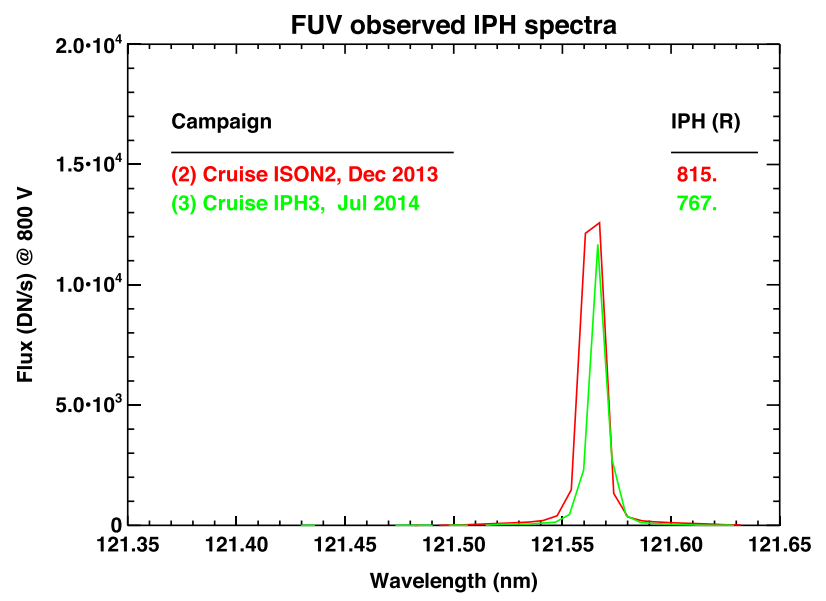

Figure 4. The IPH emission spectra observed by MAVEN IUVS-FUV. The legend on the left lists the observation campaigns. The legend on the right lists the respective radiance, in Rayleighs, determined by SWAN for these epochs, and that are corrected for SOHO-MAVEN spacecraft separation and lines of sight. The differences in line widths are attributed to the differences in viewing geometries as well as binning of the image.
The variations in peak wavelength and flux between the two campaigns are similar to and consistent with the variations in echelle observations. A summary of the FUV calibration results is listed in Table 1. A summary of operational parameters and observational details for the FUV images used in this campaign is provided in Tables $\mathrm{A} 1$ and $A 2$, respectively.

\section{Calibration Validation}

To check the consistency of the resulting calibration, measurements of Mars $\mathrm{H}$ Lyman $\alpha$ using HST made on the same day as MAVEN IUVS observations have been used. A model was used to alter the viewing geometry from that of HST's LOS to that of the IUVS observations to generate independent 


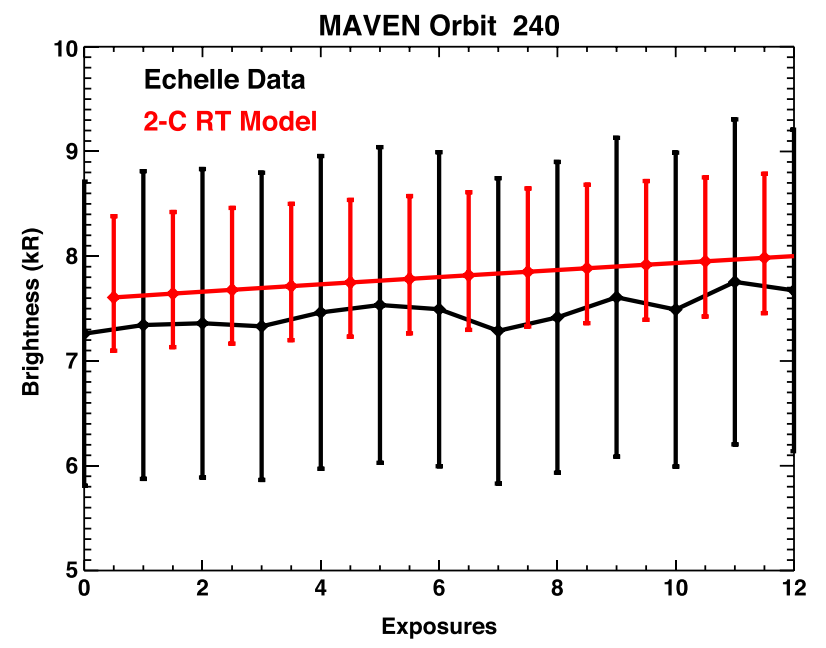

Figure 5. HST ACS-based model results of exospheric hydrogen Lyman $\alpha$ brightness as derived with a two-component radiative transfer model in red, compared with MAVEN echelle observations of exospheric hydrogen Lyman $\alpha$ in black. Exposures refer to individual images that were taken in series as part of the same observation data file from the outbound segment of MAVEN orbit 240, taken at 20:20:22 UTC. The HST and MAVEN observations were made on the same day: 12 November 2014. The vertical lines show the uncertainty in each of the RT model calibration as well as the echelle results. The RT model data points are offset by an added half of an exposure from the MAVEN data points for clarity. A $201 \times 18$ binning scheme was used, with the pixels binned $2 \times 38$ starting at a lower left corner of spectral pixel 293 and spatial pixel 316 on the full detector. Each exposure was $29.4 \mathrm{~s}$ long and was taken at $\sim 800 \mathrm{~V}$. One dark exposure is available for image reduction. More details on MAVEN IUVS echelle image reduction and uncertainties are described in the Appendix. brightness values for comparison and verification of the calibration factor used with the MAVEN data. Additionally, simultaneous FUV-mode and echelle-mode observations are used and compared by using starcalibrated values for FUV-mode data with the IPH-calibrated values for echelle-mode data.

\subsection{HST and Radiative Transfer Model}

The HST Advanced Camera for Surveys (ACS) instrument utilizes an FUV channel that has been used to image the extended hydrogen corona of Mars [Clarke et al., 2011; Avila et al., 2015]. HST made ACS observations of Mars on November 2014 at a time when MAVEN was in orbit around Mars and when the echelle instrument was imaging the sunlit disk of the planet.

A radiative transfer (RT) model, developed at Boston University, based on the model of Chaufray et al. [2008], has been used to study variability in the hydrogen corona of Mars using HST ACS observations. This RT code simulates the extended $\mathrm{H}$ corona Lyman $\alpha$ brightness at Mars and uses the observations to constrain the abundance and temperature of neutral hydrogen at the exobase. The model simulates the brightness emitted by a dual component population that includes a thermal as well as an additional suprathermal (hot) population of hydrogen atoms and is referred to as a two-component model [Bhattacharyya et al., 2015, 2016].

The RT model was used to constrain the temperature and number density of Martian exospheric hydrogen along the line of sight of a particular HST observation that overlapped with a MAVEN observation on 12 November 2014. The modeled atmosphere was then used to estimate the disk brightness of the $\mathrm{H}$ Lyman $\alpha$ emission that corresponded to the MAVEN viewing geometry along the line of sight of the IUVS echelle observations. The model assumes an isotropic and isothermal distribution of exospheric Martian hydrogen along the entirety of the path length along the LOS. The HST-based modeled brightness and the SWANcalibrated echelle brightness of Mars $\mathrm{H}$ were compared, as shown in Figure 5, in a region where the IUVS LOS solar zenith angle varied by less than $10 \%$ in order to minimize variations introduced by asymmetries in the neutral atmosphere. Both the data as well as the model show a trend of increasing brightness with time (exposure). This is due to the changing line of sight that is progressing toward larger solar zenith angles and looking through a longer column of atmosphere due to the orbital motion of the spacecraft.

The November 2014 echelle-mode observations of the Martian disk include a sequence of $\sim 30 \mathrm{~s}$ exposures. As the spacecraft moved in its trajectory, a different region of the atmosphere was being imaged. The LOS projected onto the planet surface can span over $40^{\circ}$ in latitude and solar zenith angle (SZA). For the November 2014 observation, the SZA varied between $28^{\circ}$ and $32^{\circ}$ in the subset of IUVS images used in this comparison. This subset of observations most closely approach the subsolar conditions assumed in the RT model. The reduced echelle images showed a brightness of $\sim 7.5 \pm 1.5 \mathrm{kR}$ at the disk. The RT model analysis predicted a brightness of $\sim 7.8 \pm 0.8 \mathrm{kR}$. The differences between observed and modeled values are consistent with the trend in expected differences due to proximity to subsolar conditions, where HST data taken closer 
to the subsolar point likely observe a hotter (and therefore brighter) population of $\mathrm{H}$ atoms than was observed in the MAVEN data at higher SZA. Within the observational constraints and uncertainties of each data set, there is good agreement between the model results and the observations.

\subsection{Stellar Calibration With FUV}

IUVS FUV observations have been independently calibrated by using standard stars as point sources [Snow et al., 2013]. The stellar calibration factor at the $\mathrm{H}$ Lyman $\alpha$ wavelength was determined by using observations from multiple stars made during cruise, including Alpha Crucis (HD 108248), Beta Centauri (HD 129116), and Beta Canis Major (HD 44743) [Chaffin et al., 2014; Deighan et al., 2015]. Observations made with FUV during the overlapping calibration campaigns (ISON2 and IPH3) were calibrated by using these standard stars by using the signal integrated across the echelle aperture.

The ISON2 echelle calibration campaign showed a $0.815 \mathrm{kR}$ IPH Lyman $\alpha$ emission. FUV stellar calibrated results produced a brightness of $0.814 \mathrm{kR}$ for the same campaign. The IPH3 echelle calibration campaign showed a $0.767 \mathrm{kR}$ IPH Lyman $\alpha$ emission, compared with the FUV stellar calibrated values of $0.697 \mathrm{kR}$. The calibration factors obtained by using diffuse emission IPH, for echelle and FUV, are consistent with those obtained by using standard star point-source emissions, for FUV, to within $6 \%$, on average.

The FUV calibration factor combined stellar observations and $\mathrm{H}$ corona modeling to reach an adopted calibration value [Chaffin et al., 2014]. The echelle calibration factor presented in this work agrees with the FUV adopted factor to within $10 \%$ and does not take into account any errors (systematic or otherwise) in the independently obtained FUV calibration factor.

\section{IPH Cruise Observations}

Observations of the interplanetary hydrogen emission at Lyman $\alpha$ have been made for over half a century by numerous spacecraft in orbit around Earth or the Sun [e.g., Bertaux and Blamont, 1971; Quémerais et al., 2013]. The MErcury Surface, Space ENvironment, GEochemistry, and Ranging spacecraft carried an ultraviolet-visible spectrometer that measured IPH emissions in the inner heliosphere between 0.3 and 20.6 AU during its cruise between 2009 and 2011 [McClintock and Lankton, 2007; Quémerais et al., 2014]. The two Voyager ultraviolet spectrometers and Pioneer 10 ultraviolet photometer have made measurements of the IPH through the outer regions of the heliosphere [e.g., Judge et al., 2005; Gangopadhyay et al., 2006; Quémerais et al., 2010]. Observations using the New Horizon's ALICE ultraviolet imaging spectrometer and Juno's ultraviolet spectrograph will be able to enhance the outer heliosphere data sets as well [Stern et al., 2008; Gladstone et al., 2014].

The flow of IPH entering the solar system is estimated to be $\sim 23 \mathrm{~km} \mathrm{~s}^{-1}$ [Vincent et al., 2011, 2014] along the direction of $252.2^{\circ}$ ecliptic longitude and $8.9^{\circ}$ ecliptic latitude [Quémerais et al., 1999, 2006; Lallement et al., 2010]. The distribution and characteristics of the IPH flow, as observed along the LOS of the IUVS instrument, can subsequently vary during the MAVEN spacecraft cruise due to observing geometry, the LOS velocity vector of the moving spacecraft, as well as due to solar cycle and possible interstellar variations [Ruciński and Bzowski, 1995]. The MAVEN IUVS echelle mode cruise and orbit data sets can provide models of the IPH temperature, bulk velocity, and density with data points beyond Earth's orbit over time periods consistent with the mission timeline, for the first time, at Mars' orbital distance. These data can be used to better quantify the variability in IPH properties within the solar system by providing IPH models with constraints between the orbits of Earth and Mars.

The MAVEN spacecraft was cruising at $\sim 26 \mathrm{~km} / \mathrm{s}$ relative to the Sun. For most of the cruise phase, the IUVS line of sight was varied to look near the upwind, crosswind, and downwind directions of the IPH flow ( 23 km/s). The subsequent variation in IPH emission wavelength Doppler shift approached $\sim-40 \mathrm{~km} / \mathrm{s}$ for the near-upwind measurements, $\sim-5 \mathrm{~km} / \mathrm{s}$ for the near crosswind measurements, and $\sim 35 \mathrm{~km} / \mathrm{s}$ for the near downwind measurements.

During cruise, over 20,000 minute-long integrations of the IPH were made by IUVS in the echelle mode between December 2013 and June 2014. These data have been reduced (see Appendix A for image reduction details) to derive the spectra of the interplanetary Lyman $\alpha$ emission, and then fit to a curve to derive IPH parameters of the spectra such as brightnesses, line widths, and relative Doppler shifts of the peak emission 

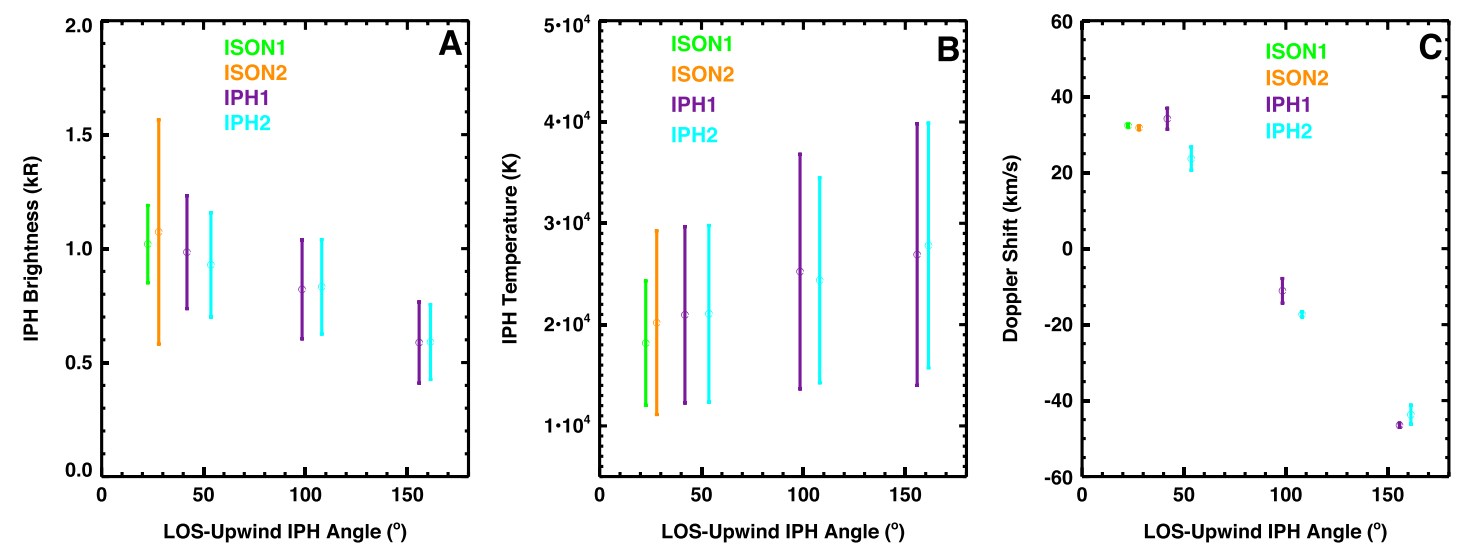

Figure 6. Properties of the IPH derived from the MAVEN IUVS echelle observations along the instrument line of sight. The data points are color coded by observation epochs: ISON1 (green), ISON2 (orange), IPH1 (purple), and IPH2 (blue). The (a) IPH brightness, (b) temperature-equivalent line width, and (c) Doppler shift are shown as a function of line of sight angle from the upwind IPH direction. A zero LOS-upwind IPH angle theoretically occurs when the instrument line of sight is most directly facing the IPH flow. The vertical lines show the standard deviation $(1 \sigma)$ about the mean shown as circles. Observation details for the data used here are given in Table 2.

wavelength. The resulting variations of the characteristics derived from the echelle cruise observations, as a function of the instrument line of sight angle from the IPH upwind direction, are shown in Figure 6.

The IPH observations used here occurred during four campaigns: ISON1 (12 December 2013), ISON2 (14-15 December 2013), IPH1 (2-18 April 2014), and IPH2 (18 April to 30 June 2014). The ISON1 and ISON2 observations occurred over 1 to 2 days and have little variation in look direction. The resulting Doppler shift along the line of sight does not vary significantly over these observations and so the IPH spectral characteristics from these campaigns have each been averaged over the respective epoch data set. The IPH1 and IPH2 campaigns spanned a longer timeline, and each had three different look directions. The IPH spectral characteristics from these look directions were averaged and shown in Figure 6 . The vertical error bars in those panels represent the $1 \sigma$ standard deviation about the mean. The results and observational details of the IPH data, shown in Figure 6, are summarized in Table 2.

On average, the MAVEN cruise echelle observations measured an IPH brightness of $\sim 0.84 \mathrm{kR}$ and a temperature of $\sim 23,000 \mathrm{~K}$ when the spacecraft was between 1 and $1.5 \mathrm{AU}$. Although the measurements made at these interplanetary distances depend on the line of sight, the general averages of the calculated IPH characteristics are consistent with previous findings for observations made near $1 \mathrm{AU}$. The Galileo spacecraft ultraviolet spectrometer observations of IPH Lyman $\alpha$ were found to vary between 0.3 and $1.2 \mathrm{kR}$ across different look directions [Ajello et al., 1994]. HST measurements using the Goddard High Resolution Spectrograph showed IPH Lyman $\alpha$ brightnesses to vary between 0.26 and $0.96 \mathrm{kR}$ with temperatures that varied between 7000 and $45,000 \mathrm{~K}$, when scanning along the IPH upwind, downwind, and cross-wind directions [Clarke et al., 1998].

Table 2. IPH-Derived Spectral Characteristics, With One Standard Deviation, From IUVS Echelle Cruise Observations Shown in Figure 6

\begin{tabular}{|c|c|c|c|c|c|}
\hline Epoch & $\begin{array}{l}\text { Average Ecliptic } \\
\text { Longitude (deg) }\end{array}$ & $\begin{array}{l}\text { Average Ecliptic } \\
\text { Latitude (deg) }\end{array}$ & $\begin{array}{l}\text { Average Doppler } \\
\text { Shift }(\mathrm{km} / \mathrm{s})\end{array}$ & $\begin{array}{c}\text { Average Brightness } \\
\qquad(\mathrm{kR})\end{array}$ & $\begin{array}{l}\text { Average Temperature } \\
\qquad\left(\times 10^{4} \mathrm{~K}\right)\end{array}$ \\
\hline ISON1 ${ }^{\mathrm{a}}$ & $242 \pm 0.740$ & $29.4 \pm 1.18$ & $32.4 \pm 0.335$ & $1.02 \pm 0.169$ & $1.82 \pm 0.614$ \\
\hline ISON2 ${ }^{\mathrm{b}}$ & $242 \pm 0.706$ & $35.3 \pm 2.22$ & $31.8 \pm 0.474$ & $1.07 \pm 0.492$ & $2.02 \pm 0.906$ \\
\hline IPH1-LOS1 ${ }^{c}$ & $80.4 \pm 0.325$ & $13.9 \pm 4.15$ & $-46.5 \pm 0.566$ & $0.59 \pm 0.178$ & $2.69 \pm 1.29$ \\
\hline IPH1-LOS2 ${ }^{\mathrm{C}}$ & $57.6 \pm 9.12$ & $72.2 \pm 3.44$ & $-11.1 \pm 3.23$ & $0.82 \pm 0.217$ & $2.52 \pm 1.16$ \\
\hline IPH1-LOS3 ${ }^{c}$ & $271 \pm 0.210$ & $47.6 \pm 4.31$ & $34.2 \pm 2.76$ & $0.98 \pm 0.248$ & $2.10 \pm 0.870$ \\
\hline IPH2-LOS1 ${ }^{d}$ & $86.6 \pm 4.64$ & $3.00 \pm 1.88$ & $-43.7 \pm 2.51$ & $0.59 \pm 0.164$ & $2.78 \pm 1.21$ \\
\hline $\mathrm{IPH}_{2}-\mathrm{LOS} 2^{\mathrm{d}}$ & $87.0 \pm 11.3$ & $62.2 \pm 1.74$ & $-17.3 \pm 0.686$ & $0.83 \pm 0.207$ & $2.44 \pm 1.01$ \\
\hline IPH2-LOS3 ${ }^{d}$ & $276 \pm 5.45$ & $59.4 \pm 2.11$ & $23.7 \pm 3.05$ & $0.93 \pm 0.229$ & $2.11 \pm 0.871$ \\
\hline
\end{tabular}

a ISON1 observations were made on 12 December 2013.

bISON2 observations were made between 14 and 15 December 2013.

cAll look directions of IPH1 observations used here were made between 3 and 14 April 2014.

${ }^{\mathrm{d}}$ All look directions of IPH2 observations used here were made between 19 and 26 April 2014. 
Table 3. FUV-Echelle Count Rate Comparison for Observations Within $2^{\circ}$ of LOS

\begin{tabular}{|c|c|c|c|c|c|c|}
\hline No. & Epoch (in chronological order) & Date & Gain (V) & FUV Int. Flux ${ }^{\mathrm{a}}$ (DN/s) $5 \mathrm{xfwhm}^{\mathrm{b}}$ & Echelle Int. Flux (DN/s) & Count Rate Ratio (FUV/ECH) \\
\hline \multicolumn{7}{|c|}{ Common Calibration Observations } \\
\hline 2 & ISON2 & Dec 2013 & 850 & 61,738 & 6,188 & 9.97 \\
\hline 3 & $\mathrm{IPH} 3$ & Jul 2014 & 800 & 18,079 & 2,499 & 7.23 \\
\hline \multicolumn{7}{|c|}{ Common Cruise Observations } \\
\hline 6 & IPH1-mode011 & Apr 2014 & 800 & 11,027 & 1,327 & 8.31 \\
\hline 7 & IPH1-mode012 & Apr 2014 & 800 & 18,658 & 2,248 & 8.30 \\
\hline \multicolumn{7}{|c|}{ Common Science Orbit Observations } \\
\hline 8 & Orbits 500 & Jan 2015 & 800 & 127,643 & 20,401 & 6.26 \\
\hline 9 & Orbits 1600 & Jul-Aug 2015 & 800 & 34,842 & 4,290 & 8.12 \\
\hline 4 & Mars Orbit & Oct 2015 & 800 & 30,750 & 4,062 & 7.57 \\
\hline
\end{tabular}

${ }^{a}$ Includes a correction factor to account for different regions on the detector used to extract the FUV signal between spatial rows 377 and 505 ( 130 rows), to the echelle signal integrated spatially across the aperture between spatial rows 346 and 535 ( 190 rows).

${ }^{b}$ Five FWHM about the peak FUV signal were used as spectral integration limits.

More recently, SWAN observations measured the IPH Lyman $\alpha$ emission and derived brightnesses that varied between 0.4 and $1.4 \mathrm{kR}$ and temperatures that varied between 5000 and 30,000 $\mathrm{K}$ along different look directions [Quémerais et al., 2008].

Analyzing the temperature and velocity variability of the IPH flow along the look direction has been previously done [Quémerais et al., 2006]. The temperature was found to be coolest $(\sim 14,000 \mathrm{~K})$ when calculated from upwind measurements and warmest ( 18,000 K) when calculated from downwind measurements. The IUVS echelle IPH temperature findings are consistent with the observed trend of increasing temperature with LOS-upwind angle. The echelle data-derived average IPH temperature varies from $\sim 18,000 \mathrm{~K}$ for near upwind measurements to $\sim 28,000 \mathrm{~K}$ for near downwind measurements. The temperature differences between previous works and the present work may be due to some systematic differences in temperature calculation methods and are currently being investigated.

The LOS velocity, from the Sun's reference frame, was found to vary between $\sim-22 \mathrm{~km} \mathrm{~s}^{-1}$, when viewed purely upwind (at an LOS-IPH upwind angle of $0^{\circ}$ ), and $\sim-26 \mathrm{~km} \mathrm{~s}^{-1}$ when viewed downwind (at an LOSIPH upwind angle of $180^{\circ}$ ), from measurements made in 1996 and 1997 [Quémerais et al., 2006]. The findings for velocity distributions, from the MAVEN spacecraft frame of reference, were found to vary between $\sim 32 \mathrm{~km} \mathrm{~s}^{-1}$ at an LOS-IPH upwind angle of $\sim 23^{\circ}$ and $-47 \mathrm{~km} \mathrm{~s}^{-1}$ at an LOS-IPH angle of $161^{\circ}$. The IUVS echelle-derived IPH properties, shown in Figure 6, use the spacecraft frame of reference. MAVEN was moving at $\sim 25 \mathrm{~km} \mathrm{~s}^{-1}$ with respect to the Sun, and the spacecraft velocity component along the line of sight contributed the absolute value of velocity variability along the LOS. When correcting for that, our velocity shift findings are consistent with previous measurements.

The ongoing analysis of in-orbit echelle observations shows opportunities where the IPH emission was sufficiently Doppler shifted from the Mars H emission to be resolved. Future observing efforts will be dedicated to examining these opportunities to increase the coverage of IPH spectral properties at Mars' orbital location over the timeline of the MAVEN mission. The variability of the IPH spectral brightness and temperature values, calculated from the MAVEN echelle data during cruise and at orbit, would provide valuable constraints to models of the IPH. In future work, more IUVS IPH observations will be analyzed to map the LOS-specific derived spectral properties. These properties could then be used with models to interpret the variability of interplanetary hydrogen along single lines of sight and will be useful for deriving dynamical properties of the interplanetary and interstellar medium.

\section{Results and Discussion}

The echelle and FUV modes of the MAVEN IUVS instrument have been calibrated the hydrogen Lyman $\alpha$ wavelength, for similar regions of the detector, using simultaneous SWAN observations of diffuse IPH emission. The derived echelle calibration factor was found to be stable throughout the five cross-calibration campaigns used in this work. The results from the IUVS-SWAN cross-calibration analysis are summarized in Table 1 for echelle-mode observations and for FUV-mode observations. The average calibration factor 
determined from five epochs for the echelle is $29.8 \pm 5.69$ photons/s/kR ( $3543 \pm 709 \mathrm{DN} / \mathrm{s}$ at $800 \mathrm{~V}$ Gain) at the hydrogen Lyman $\alpha$ wavelength. The average calibration factor determined from two epochs for FUV is $252 \pm 54$ photons/s/kR $(29,988 \pm 6426 \mathrm{DN} / \mathrm{s}$ at $800 \mathrm{~V}$ Gain $)$ at the hydrogen Lyman $\alpha$ wavelength. For this set of overlapping calibration campaign observations for the two modes, the average FUV-mode to echelle-mode count rate ratio is 8.46 .

Observations outside of the calibration campaigns were used to monitor the instrument sensitivity at Lyman $\alpha$, as constrained by similar lines of sight, to within a few degrees. The observations used to examine instrument degradation began during cruise, included calibration campaigns, and continued throughout the science phase of the mission through August 2015 (orbit 1694). Table 3 summarizes the results that pertain to instrument degradation obtained from these observations. Epochs (2) and (3) include the observations used for instrument calibration, as described in Table 1. Epochs (6) and (7) refer to observations made during cruise "IPH1"-type observations that included imaging the IPH along multiple look directions. Modes 011 and 012 of IPH1 observations refer to two specific look directions in the echelle data files that had common pointing to FUV observations. Observations made when MAVEN was orbiting Mars were also used to monitor instrument degradation. Epoch (4) includes a data set where both IPH and Mars H Lyman $\alpha$ brightnesses observed in the echelle mode were combined and compared with FUV-observed brightnesses, along similar lines of sight, since the FUV cannot resolve the contributions of each source. Epochs (5) and (6) were observations made during the inbound segment of the spacecraft orbit. As in epoch (4), the IPH Lyman $\alpha$ contributions in epoch (5) and (6) echelle observations were included with Mars $\mathrm{H}$ to compare with similarly pointed FUV observations.

No instrument degradation was noted when different mode IUVS observations were compared at various epochs throughout cruise and during orbit. The average ratio between FUV-mode and echelle-mode count rates is 7.97 when averaged over all the comparative observations (listed in Table 3). The differences in echelle integrated flux between Tables 1 and 3 for epoch (4) are due to the inclusion of Mars $\mathrm{H}$ in the latter.

The IUVS FUV-mode calibration factor at the same wavelength was consistent with the expected sensitivity for that mode, and the calibrated results were independently and successfully validated with HST observations and standard stars. HST measurements and modeling results of exospheric hydrogen at Mars show that there is good agreement (to within 4\%) with the simultaneous observations made by MAVEN IUVS echelle. IUVS FUV observations of in-cruise IPH, calibrated independently using stars, agreed with simultaneous echelle observations (to within $~ 6 \%$ ).

The distributions of interplanetary hydrogen brightness and temperature variations are provided, for the first time, at the orbital distances covered by MAVEN during cruise. The derived IPH properties can provide validation points for models of the IPH and therefore improve interpretation of dynamics within the inner heliosphere. These characteristics can be applied toward interpretations of the dynamics of the interaction between the interstellar medium and its galactic counterpart at the boundaries of our solar system [e.g., Quémerais et al., 2013; Lallement et al., 2011].

Using the calibration factors described in this work will be useful for analyzing the absolute fluxes of hydrogen, deuterium, oxygen, and other species FUV emissions detected by the echelle mode of the MAVEN IUVS instrument. An analysis of the resulting brightness values and the ratios of specific resolved lines will allow for improved insights into the evolution of water in the atmosphere of Mars.

\section{Appendix A}

IUVS echelle data files are available in the NASA public data archives as fits files. Each of these data files is typically composed of several images. Each image in the same data file is referred to as an exposure or frame. The number of exposures in a data file varies with different observation type. The integration time for exposures also varies with each observation type; however, the integration time is the same for all exposures of a given data file.

\section{A1. Observation Details}

Operational specifications of the echelle-mode and FUV-mode observations used for the SWAN crosscalibration data analysis are specified in Table A1. The properties of the detector used for the observations 


\begin{tabular}{|c|c|c|c|c|c|c|c|c|c|}
\hline No. & Epoch & $\begin{array}{c}\text { Size } \\
\text { (spectral } \times \text { spatial) }\end{array}$ & $\begin{array}{c}\text { Binning } \\
\text { (spectral } \times \text { spatial) }\end{array}$ & $\begin{array}{l}\text { Low } \\
\text { Corner }(x, y)\end{array}$ & $\begin{array}{c}\text { High } \\
\text { Corner }(x, y)\end{array}$ & Exposures & Gain (V) & $\begin{array}{l}\text { Integration } \\
\text { Time (s) }\end{array}$ & $\begin{array}{l}\text { Darks per Light } \\
\text { (images } \times \text { exposures) }\end{array}$ \\
\hline \multicolumn{10}{|c|}{ Echelle } \\
\hline 1 & ISON1 & $201 \times 287$ & $2 \times 3$ & 138,100 & 540,962 & 36 & 850 & 60 & $2 \times 1$ \\
\hline 2 & ISON2 & $201 \times 287$ & $2 \times 3$ & 138,100 & 540,962 & 36 & 850 & 60 & $2 \times 1$ \\
\hline 3 & $\mathrm{IPH} 3$ & $1024 \times 1024$ & $1 \times 1$ & 0,0 & 1023,1023 & 1 & 800 & 60 & $1 \times 1$ \\
\hline 4 & Mars Orbit (Oct 2015) & $201 \times 287$ & $2 \times 3$ & 138,100 & 540,962 & 60 & 800 & 60 & $2 \times 3$ \\
\hline 5 & Mars Orbit (Feb 2016) & $201 \times 287$ & $2 \times 3$ & 138,100 & 540,962 & 30 & 800 & 60 & $3 \times 3$ \\
\hline \multicolumn{10}{|c|}{ FUV } \\
\hline 2 & ISON2 & $128 \times 128$ & $8 \times 8$ & 0,0 & 1023,1023 & 36 & 850 & 60 & $2 \times 1$ \\
\hline 3 & $\mathrm{IPH} 3$ & $512 \times 256$ & $2 \times 4$ & 0,0 & 1023,1023 & 1 & 800 & 60 & $2 \times 1$ \\
\hline
\end{tabular}

${ }^{\mathrm{a}}$ The spectral resolution of the echelle instrument is $\sim 15000$ at hydrogen Lyman $\alpha$.

in these epochs (with spectra shown in Figures 2 and 3) are specified. The size of the image downloaded from the spacecraft is given in binned pixels. Each binned pixel combines the photon counts from a number of columns (spectral elements) and rows (spatial elements) of the full (unbinned) $1024 \times 1024$ pixel detector. The starting point, on the full-frame detector, where the binning begins and ends is described as low corner and high corner coordinates. From these specifications, one can determine the subset of the full detector that has been used to make the observations. The number of exposures of each observation type is provided, as well as the voltage gain across the detector, and the length of the integration time of the exposures for each of the observation types. The number of dark images and exposures available for each epoch's observations are also listed.

The observation details describing the LOS of the calibration observations are summarized in Table A2. The epochs described in Figures 2 and 3 are listed for reference. The following properties of the observational line of sight are listed: right ascension (RA), declination (DEC), and solar zenith angle (SZA). These properties vary from the first exposure of the first image used to the last exposure of the last image of each epoch and so the range of values for these quantities is listed. For observations made at Mars, additional observational properties of the line of sight are given, specifically, the range of planetary latitude and longitude of the line of sight.

\section{A2. Echelle Image Reduction}

Procedures for operationally optimizing IUVS measurements have evolved throughout the mission resulting in variations in the characteristics of the observations. Some of these characteristics are the number of dark images taken for each light image, the number of exposures in dark and light images, the integration time of images, binning scheme, the portion of the full detector used, and voltage gain. These variations are treated consistently for all types of observations in the image reduction process. To reduce an echellogram from its raw format (level1a file) to facilitate calculation of emission line absolute brightness, a series of techniques in the form of a pipeline was used for each observation.

\begin{tabular}{|c|c|c|c|c|c|c|}
\hline No. & Epoch & $\begin{array}{c}\text { LOS RA Range } \\
\text { (deg) }\end{array}$ & $\begin{array}{c}\text { LOS DEC Range } \\
\text { (deg) }\end{array}$ & $\begin{array}{c}\text { LOS SZA Range } \\
\text { (deg) }\end{array}$ & $\begin{array}{l}\text { LOS Latitude } \\
\text { Range (deg) }\end{array}$ & $\begin{array}{r}\text { LOS Longitude } \\
\text { Range (deg) }\end{array}$ \\
\hline \multicolumn{7}{|c|}{ Echelle } \\
\hline 1 & ISON1 & $243-247$ & $4.3-16$ & & & \\
\hline 2 & ISON2 & $244-249$ & $8.2-20$ & & & \\
\hline 3 & IPH3 & $153-158$ & $79-80$ & & & \\
\hline 4 & $\begin{array}{c}\text { Mars Orbit (Oct } \\
\text { 2015) }\end{array}$ & $227-228$ & -21 to -9.4 & $100-134$ & $23-59$ & $225-245$ \\
\hline 5 & $\begin{array}{c}\text { Mars Orbit (Feb } \\
\text { 2016) }\end{array}$ & $22-54$ & $3.1-9.9$ & $102-107$ & -83 to -74 & $187-231$ \\
\hline \multicolumn{7}{|c|}{ FUV } \\
\hline 2 & ISON2 & $246-247$ & $13-17$ & & & \\
\hline 3 & IPH3 & $161-165$ & $79-80$ & & & \\
\hline
\end{tabular}


In general, the image reduction pipeline includes dark subtraction, image filtering to account for energetic particle impacts to the detector that affect one or more pixels, image filtering to account for temporarily or permanently affected pixels on the detector, removal of background signal as well as scattered light, and correcting for observation-dependent nuances in the optical system (such as voltage gain across the detector and binning scheme). Each of these processes is described in more detail below.

\section{A2.1. Dark Subtraction}

An echelle dark image is an exposure taken with the same cadence as a light image and that is close in time to the light exposure(s). A dark exposure is taken by closing the electronic shutter in order to record and correct for the dark current in the electronics system. Early in the mission, a single dark image with one exposure was taken for each light image, where a light image can have one or (more commonly) multiple exposures. Analysis of these images showed that there were variations in the first exposure of any type of observation (dark or light) that did not appear in the second exposure. To correct for these variations, two exposures were taken in each dark echelle image. The first dark exposure was paired with the first light exposure, and the second dark exposure was paired with all the remaining light exposures. The first step of the image reduction pipeline was to consistently utilize all available dark exposures and subtract them from the light image exposures of that observation set.

\section{A2.2. Energetic Particle Effects}

The dark-subtracted images are next processed through a filter to remove energetic particle effects that impact random regions of the detector by utilizing each pixel element of the light image exposures. For an image with $201 \times 287$ binned pixels and 36 exposures, for example, the energetic particle filter would isolate the array of each individual pixel across all 36 exposures and replace any pixel value that lay outside 2 standard deviations of the median of the 36 pixel values by the median DN value. This was repeated for all pixels and was found to consistently and adequately remove random impacts to the detector for multiple binning schemes and exposures used.

This filtering process occurred after dark current subtraction and therefore accounted for energetic particle events that may have affected the dark exposures as well as the light ones. In cases where there were only single light exposures available, the entire set of available light images of a given observation set was utilized for the median filtering, since these images had consistent characteristics and were observed close in time.

\section{A2.3. Hot Pixel Effects}

Several pixel elements on the detector were temporarily or permanently offset from the rest due to electronics or other issues. A second filter was used across the resulting image set to account for these outlying (aka hot) pixels. This filter was applied to each exposure by scanning a $7 \times 7$ square region centered around each individual pixel of an exposure and replacing the center pixel with the median of the $7 \times 7$ square region if that pixel was outside of 3 standard deviations of the median of that region. This process was repeated for each pixel in each exposure and consistently removed hot pixels for various binning schemes.

\section{A2.4. Background Subtraction}

The echelle images showed a uniform background signal that results from a combination of thermal noise and energetic particles hitting the detector. Stray light can also theoretically affect the detected signal, but the echelle channel has not made such detections. Calculating an average background signal from each exposure and then removing that signal from the full image of each exposure are done to account for these effects. Emission-free regions on the FUV detector were selected for estimating the average background signal. These regions are typically chosen from rows that lay spatially below and above the rows where the hydrogen $121.567 \mathrm{~nm}$ emission is projected onto the detector. The count rates from the rows without emissions were then averaged to form a representative background signal per row.

The row-averaged background signal was consistently calculated for multiple binning schemes and subsequently used in the pipeline to account for background contributions by removing them from each row of the emission region. In cases where the observation includes emissions other that $\mathrm{H}$ and $\mathrm{D}$ (that are centered on the full-frame detector), then different rows that are close to those of the emission lines were used instead to generate a different row-averaged background.

A2.5. Spatial Scattered Light

By design, the emission region spatially spans $\sim 150$ rows on the full detector. The main order of $\mathrm{H}$ Lyman $\alpha$ emission is centered on the full-frame detector. As shown in Figure 1b, the observed echellogram shows the 
hydrogen emissions to span a wider region (spatially and spectrally) than the theoretically predicted allotment due to scattered light from the echelle grating appearing on the detector.

The integration limits of each bright emission line on the detector were spatially adjusted in order to include spatially scattered light. The main order of $\mathrm{H}$ Lyman $\alpha$, for example, was effectively found to span $\sim 190$ rows (up from the theoretical 130). On the full-frame detector, this was found to be between rows 346 and 535 .

The coarser resolution IUVS FUV mode observes the same emission lines differently, with the $\mathrm{H}$ Lyman $\alpha$ spanning more spatial rows than in echelle mode. For consistency in echelle to FUV comparisons, the aperture-equivalent rows were used to integrate the FUV hydrogen $121.567 \mathrm{~nm}$ as those used for echelle. These rows covered the same field of view in each mode.

\section{A2.6. Spectral Scattered Light}

A line spread function (LSF) has been generated from averaging the dispersion response of multiple laboratory observations made during instrument design and implementation. The wings of the LSF accurately account for the spectral scattered light response of the instrument to multiple emission lines. Mars emissions detected by the echelle mode (including $\mathrm{H}, \mathrm{D}, \mathrm{O}$, and others) are well fit to the instrument line spread function (LSF). The broader IPH emission that is used for calibration is best fit to the instrument LSF convolved with Voigt curve, LSFaVoigt $(T)$, where $T$ is a temperature representative of the width of IPH emission. The Voigt profile is used to combine the broadening of a Gaussian distribution as well as a Lorentzian. The LSF is chosen to best represent the spread in the wings on each side of the emission peak.

At this sequential stage of the pipeline, the images have been reduced and used to produce a spectrum for each emission line detectable above the background noise. For Mars emissions, the resulting spectra were fit to the instrument LSF in order to account for spectral scattered light in the dispersion direction. The best fit curve was determined from iteratively varying the LSF peak flux and wavelength to minimize the chi-square $\left(\chi^{2}\right)$ difference between the fit and the data.

For IPH emissions, the resulting reduced spectrum was best fit to an LSF-Voigt convolved profile to account for the broader IPH line. The best fit curve was determined from iteratively varying the peak of the line profile, the wavelength of the peak of the emission, and the width of the Voigt profile (i.e., the IPH temperature) to minimize the $\chi^{2}$ difference between the fit and the data. The temperature of the IPH varied between $17,000 \mathrm{~K}$ and $41,000 \mathrm{~K}$, consistent with observations of interplanetary hydrogen as viewed through different look directions [Bertaux et al., 1977; Vincent et al., 2014].

\section{A2.7. Signal Integration}

The resulting best fit curve was integrated to within 5 full width at half maximum (FWHM) units on each side of the peak for IPH emissions and 12 FWHM units on each side of the peak of Mars species emissions. This was done to capture $88 \%$ of the total signal as optimized by binning restrictions, spectral range, and curve used for fitting (LSF for Mars emissions or LSF-Voigt for IPH emission). A correction factor of $\sim 1.13$ is applied to account for the remaining $12 \%$ of the scattered light of the signal that was excluded from the integration limits. With $100 \%$ of the signal accounted for, comparisons to SWAN-derived values of IPH brightness or HST-derived values of Mars coronal $\mathrm{H}$ could be made.

\section{A2.8. Other Adjustments}

The echelle instrument can detect oxygen solar resonance triplet $(130.217,130.486$, and $130.603 \mathrm{~nm})$ and oxygen forbidden doublet $(135.560$ and $135.851 \mathrm{~nm}$ ) emission lines as well as FUV emissions from other species such as $\mathrm{N}, \mathrm{CO}$, and $\mathrm{C}^{+}$in the atmosphere of Mars [McClintock et al., 2014]. These emission lines fall on different regions of the detector than hydrogen and deuterium. Adjustments to the calibration factor are made to account for the blaze function as well as for sensitivity variability across the detector.

The echelle pipeline produced integrated fluxes from Martian atmospheric FUV emitting species as well as from IPH that represent the total photon count rate received at the detector from those emissions. The next step in the calibration process, as described below, was to obtain a calibration factor to convert the integrated count rate into absolute brightness in kR.

\section{A3. FUV Image Reduction}

The differences between using FUV mode and echelle mode to cross calibrate the instrument at the $\mathrm{H}$ Lyman $\alpha$ wavelength dictate that different image reduction techniques be used to derive emission brightness 
values. However, the same pipeline developed for echelle observations was adopted for FUV to maintain consistency in the methodologies used to derive integrated count rates of $\mathrm{PH}$, with minor adjustments made to accommodate for the background noise calculation and line profile shape observed in the coarserresolution images.

The FUV exposures project the aperture spatially across most of the detector, resulting in there being too few rows above and below the signal to calculate an average background. To remove the background noise, the off-emission regions redward and blueward of several FWHM of the peak were used to calculate a background signal and then subtracted from the integrated spectrum. The FUV-mode observations also do not fit the echelle-specific LSF. The emission lines are relatively narrow with minimal scattering in the dispersion direction and can therefore be simply integrated across few FWHM on each side of the peak to calculate the full signal count rate.

To get an integrated H Lyman $\alpha$ signal from an FUV exposure that is comparable to that obtained from an echelle exposure, for similar LOS pointing conditions, different regions of the detector were used for each mode to capture count rates. The projected image of the $3 \mathrm{~mm}$ echelle aperture on to the $1024 \times 1024$ detector is 130 vertical pixels (rows) [McClintock et al., 2014]. Instrumental aberrations blur the vertical extent of the aperture. Inflight observations have shown the FWHM of the projected aperture image to be 130 pixels with emissions reaching several rows above and below the edges of the aperture projection, demonstrated in Figure 1. As a result, 190 rows on the detector, centered at the $\mathrm{H}$ Lyman $\alpha$ line center location, are coadded to capture all the light passing through the aperture to obtain the full count rate in echelle mode exposures. In FUV mode, the aperture-equivalent 130 vertical pixels (rows) were used to integrate the corresponding FUV signal. Specific row numbers used are described in footnote (a) of Table 3.

\section{A4. Uncertainties}

Known uncertainties have been accounted for in the echelle and FUV channel cross-calibration procedures. Comments on systematic uncertainties are reserved and assumed to lie within the known sources of error discussed below.

\section{A4.1. SWAN Model}

In the five calibration campaigns to date, SWAN IPH observations overlapped with those of MAVEN IPH observations during early cruise epochs (a) and (b), when the spacecraft was close to $1 \mathrm{AU}$. These measurements required the least adjustments due to the proximity of MAVEN to SOHO and due to the similarity of the MAVEN-IUVS and SOHO-SWAN lines of sight. The remaining campaigns occurred when MAVEN was farther away from Earth (and subsequently, from SOHO). To account for the separation in location of the two spacecraft, a correction factor was applied based on a model of the interplanetary medium that accounts for the IPH cavity in the solar system and its variation with respect to observing geometry [Quémerais et al., 2008; Izmodenov et al., 2013]. The errors introduced in this methodology are within $10 \%$.

\section{A4.2. Spectral Line Curve Fits}

Mars emission lines were fit to the instrument LSF. The broader IPH lines were fit to a curve that is a convolved by-product of the instrument LSF and a Voigt profile. Adjustments to the spectral location, peak, and width of the curve were made iteratively to reach a minimum $\chi^{2}$ difference between the observed spectrum and the fit. The uncertainties introduced by using these best fit curves are within $10 \%$.

The variation of the peak emission due to thermal properties of the detector was not taken into account. These effects do not affect the calibration results described in this work. The effects of these thermal shifts in the dispersion direction are being investigated for subsequent impact on the calculated IPH Doppler shift distribution.

\section{A4.3. Detector Noise Levels}

The observed spectra are reduced by using the pipeline described in section 2 . Anywhere from a dozen to a few hundred reduced images were coadded to improve the signal-to-noise ratio. The signal for all of the campaigns used here is strong enough to be clearly detected above the noise and is dominated by Poisson statistics. The uncertainty resulting from this process is within $2 \%$.

\section{A4.4. Count Rate Integration}

The best fit curve was used to derive the integrated count rate of the Mars species as well as IPH emissions. Each observation campaign had variations in the binning scheme that captured a subset or entirety of the full 
detector array. These varying spectral ranges resulted in emission spectra that were fully captured (spectrally) in only one of the five campaigns that utilized the entire detector and only partially in the remaining four campaigns that used spectral subsets of the detector. As a result, the integrated signal was chosen to be the sum of the count rates under the best fit curve (per spectral unit) to within a number of full width at half maximums (FWHMs) on each side of the emission peak.

As described in section A2.7, the same percentage of total signal under each of the curves is used for cross calibration. To account for variations due to binning schemes and spectral range in each observation, symmetry assumptions were invoked and a corrective factor was included in the calibration. Since no echelle observations capture an infinite spectral range, and since the scattered light in the detector never falls down to the background noise level in echellograms, there are undersampling errors that are expected to contribute to $\sim 12 \%$ of the upper limit of the calibration factor.

\section{A4.5. Propagation of Errors}

The uncertainties discussed above in determining the calibration factor derived from observing the IPH using echelle and SWAN are mathematically propagated. Assuming that all sources of uncertainty are uncorrelated, the total uncertainty introduced in the calibration factor determination is within $20 \%$.

\section{A4.6. Noise Calculations}

The uncertainty due to noise on the detector was determined from the reduced spectrum using the following:

$$
\sigma_{\text {Poisson }}=\sqrt{\text { Noise }^{2}+\text { Signal }^{2}}
$$

The Poisson noise was calculated by using $\sqrt{N}$ statistics where $N$ is the number of counts (photons) in an emission. The IPH and Mars $\mathrm{H}$ emissions were bright enough to show a reliable signal well above the noise in each observation used.

Over 20 random regions on the detector, spectrally well separated from the main emission, were sequentially fit to an LSF to derive the uncertainty of using the best fit on the emission. The uncertainty due to detector noise in the fits was then determined from the following:

$$
\sigma_{\text {Noise }}=\frac{\sqrt{\sum^{N} \sigma_{\text {Noise }}^{2}}}{N_{\text {Noise fits }}}
$$

The uncertainties generated due to using the best fit curve was determined from the following:

$$
\sigma_{\text {fit }}=\sqrt{\frac{\sum(\text { data }- \text { fit })^{2}}{N_{\lambda}}}
$$

where data is the count rate from the observation, fit is the count rate from the best fit LSF, and $N$ is the number of data points.

Acknowledgments

This work was funded by NASA contract 1000320450 from the University of Colorado to Boston University. O.K. is supported by RFBR grant 16-52-16008CNRS_a. J.-Y.C. is supported by the Centre national d'Etudes Spatiales. The MAVEN IUVS data used in the calibration and data analysis described in this manuscript are available on the NASA Planetary Data System (http:// atmos.nmsu.edu/data_and_services/ atmospheres_data/MAVEN/calib.html). Data provided by the SOHO SWAN team for this work are provided in Table 1. The lead author would like to thank the Editor, reviewers, and the coauthors for their careful scrutiny of the manuscript that helped improve the presentation of this work.

\section{References}

Ajello, J., W. Pryor, C. Barth, C. Hord, A. Stewart, K. Simmons, and D. Hall (1994), Observations of interplanetary Lyman- $\alpha$ with the Galileo Ultraviolet Spectrometer: Multiple scattering effects at solar maximum, Astron. Astrophys., 289, 283-303.

Avila, R., et al. (2015), ACS Instrument Handbook Version 4.0, STScl, Baltimore.

Bertaux, J. L., and J. E. Blamont (1971), Evidence for a source of an extraterrestrial hydrogen Lyman-alpha emission, Astron. Astrophys., 11, 200-217.

Bertaux, J. L., J. E. Balmont, E. N. Mironova, V. G. Kurt, and M. C. Bourgin (1977), Temperature measurement of interplanetary-interstellar hydrogen, Nature, 270, 156-158, doi:10.1038/270156a0.

Bertaux, J. L., et al. (1995), SWAN: A study of solar wind anisotropies on SOHO with Lyman alpha sky mapping, Sol. Phys., 162, 403-439, doi:10.1007/BF00733435.

Bhattacharyya, D., J. T. Clarke, J.-L. Bertaux, J.-Y. Chaufray, and M. Mayyasi (2015), A strong seasonal dependence in the Martian hydrogen exosphere, Geophys. Res. Lett., 42, 8678-8685, doi:10.1002/2015GL065804.

Bhattacharyya, D., J. T. Clarke, J.-L. Bertaux, J.-Y. Chaufray, M. Mayyasi (2016), Analysis and modeling of remote observations of the Martian hydrogen exosphere, Icarus, doi:10.1016/j.icarus.2016.08.034, in proof.

Chaffin, M. S., J.-Y. Chaufray, I. Stewart, F. Montmessin, N. M. Schneider, and J.-L. Bertaux (2014), Unexpected variability of Martian hydrogen escape, Geophys. Res. Lett., 41, 314-320, doi:10.1002/2013GL058578.

Chaffin, M. S., et al. (2015), Three-dimensional structure in the Mars H corona revealed by IUVS on MAVEN, Geophys. Res. Lett., 42, 9001-9008, doi:10.1002/2015GL065287.

Chaufray, J.-Y., J.-L. Bertaux, F. LeBlanc, and E. Quemérais (2008), Observation of the hydrogen corona with SPICAM on Mars Express, Icarus, 195, 598-613, doi:10.1016/j.icarus.2008.01.009. 
Chaufray, J.-Y., F. LeBlanc, E. Quémerais, and J.-L. Bertaux (2009), Martian oxygen density at the exobase deduced from O I $130.4 \mathrm{~nm}$ observations by spectroscopy for the investigation of the characteristics of the atmosphere of Mars on Mars Express, J. Geophys. Res., 114, E02006, doi:10.1029/2008JE003130.

Chaufray, J.-Y., et al. (2015), Variability of the hydrogen in the Martian upper atmosphere as simulated by a 3D atmosphere-exosphere coupling, Icarus, 245, 282-294, doi:10.1016/j.icarus.2014.08.038.

Clarke, J. T., R. Lallement, J.-L. Bertaux, H. Fahr, E. Quémerais, and H. Scherer (1998), HST/GHRS observations of the velocity structure of interplanetary hydrogen, Astrophys. J., 499, 482-488, doi:10.1086/305628.

Clarke, J. T., J.-Y. Chaufray, J.-L. Bertaux, G. R. Gladstone, E. Quémerais, and J. K. Wilson (2011), HST observations of the Martian hydrogen corona, The Fourth International Workshop on the Mars Atmosphere: Modeling and observation, held 8-11 February, 2011, in Paris, France, p. 388-388, 2011mamo.conf..388C

Clarke, J. T., J.-L. Bertaux, J.-Y. Chaufray, G. R. Gladstone, E. Quémerais, J. K. Wilson, and D. Bhattacharyya (2014), A rapid decrease of the hydrogen corona of Mars, Geophys. Res. Lett., 41, 8013-8020, doi:10.1002/2014GL061803.

Clarke, J. T., et al. (2017), Variability of D and H in the Martian upper atmosphere observed with the MAVEN IUVS echelle channel, J. Geophys. Space Physics, doi:10.1002/2016JA023479, in press.

Deighan, J., et al. (2015), MAVEN IUVS observation of the hot oxygen corona at Mars, Geophys. Res. Lett., 42, 9009-9014, doi:10.1002/ 2015 GL065487.

Gangopadhyay, P., V. Izmodenov, M. Gruntman, and D. Judge (2006), Voyager 1, Voyager 2, and Pioneer 10 Ly $\alpha$ data and their interpretation, Astrophys. J., 637, 786-790, doi:10.1086/498444.

Gladstone, G., et al. (2014), The ultraviolet spectrograph on NASA's Juno mission, Space Sci. Rev., doi:10.1007/s11214-014-0040-z.

Harris, W., J. Clarke, J. Caldwell, P. Feldman, B. Bush, D. Cotton, and S. Chakrabarti (1993), High resolution ultraviolet spectrograph for sounding rocket measurements of planetary emission line profiles, Opt. Eng., 32, 3016, doi:10.1117/12.149165.

Izmodenov, V. (2006), Early concepts of the heliospheric interface: $\mathrm{H}$ atoms, in The Physics of the Heliospheric Boundaries, ISSI Sci Rep No. 5, edited by V. Izmodenov and R. Kallenbach, pp. 45-65, ESA-ESTEC, Paros.

Izmodenov, V., O. Katushkina, E. Quémerais, and M. Bzowski (2013), Distribution of interstellar hydrogen atoms in the heliosphere and backscattered solar Lyman- $\alpha$, in Cross-Calibration of Far UV Spectra of Solar System Objects and the Heliosphere, edited by E. Quémerais, M. Snow, and R.-M. Bonnet, pp. 7-65, Springer, New York.

Jakosky, B. (2015), MAVEN explores the Martian upper atmosphere, Science, 360, 6261, doi:10.1126/science.aad3443.

Judge, D., V. Izmodenov, M. Gruntman, and P. Gangopadhyay (2005), Interpretation of Pioneer 10 heliospheric Ly $\alpha$ glow data obtained beyond $30 \mathrm{AU}$, Astrophys. Space Sci. Trans., 1, 29-34, doi:10.5194/astra-1-29-2005.

Lallement, R., E. Quémerais, D. Koutroumpa, J.-L. Bertaux, S. Ferron, W. Schmidt, and P. Lamy (2010), The interstellar H flow: Updated analysis of SOHO/SWAN data, AIP Conf. Proc. Twelfth Int. Solar Wind Conf., 1216(p), 555-558, doi:10.1063/1.3395925.

Lallement, R., E. Quémerais, J.-L. Bertaux, B. Sandel, and V. Izmodenov (2011), Voyager measurements of Hydrogen Lyman- $\alpha$ diffuse emission from the Milky Way, Science, 334, 1665-1669, doi:10.1126/science.1197340.

McClintock, W. E., and M. R. Lankton (2007), The Mercury Atmospheric and Surface Composition Spectrometer for the MESSENGER mission Space Sci. Rev., 131, 481-521.

McClintock, W., et al. (2014), The Imaging Ultraviolet Spectrograph (IUVS) for the MAVEN mission, Space Sci. Rev., doi:10.1007/s11214-0140098-7.

Quémerais, E. (2000), Angle dependent partial frequency redistribution in the interplanetary medium at Lyman- $\alpha$, Astron. Astrophys., 358 , 353-367.

Quémerais, E., J.-L. Bertaux, R. Lallement, M. Berthe, E. Kyrölä, and W. Schmidt (1999), Interplanetary Lyman $\alpha$ line profiles derived from SWAN/SOHO hydrogen cell measurements: Full-sky velocity field, J. Geophys. Res., 104(A6), 12,585-12,603, doi:10.1029/1998JA900101.

Quémerais, E., R. Lallement, J.-L. Bertaux, D. Koutroumpa, J. Clarke, E. Kyrölä, and W. Schmidt (2006), Interplanetary Lyman- $\alpha$ line profiles: Variations with solar activity cycle, Astron. Astrophys., 445, 1135-1142, doi:10.1051/0004-6361:20065169.

Quémerais, E., V. V. Izmodenov, D. Koutroumpa, and Y. Malama (2008), Time dependent model of the interplanetary Lyman $\alpha$ glow: Applications to the SWAN data, Astron. Astrophys., 488, 351-359, doi:10.1051/0004-6361:20078204.

Quémerais, E., R. Lallement, J.-L. Bertaux, B. R. Sandel, V. V. Izmodenov, and Y. Malama (2010), Ultraviolet glow from the hydrogen wall, Astrophys. J., 711, 1257-1262, doi:10.1088/0004-637X/711/2/1257.

Quémerais, E., M. Snow and R.-M. Bonnet (2013), Cross-Calibration of Far UV Spectra of Solar System Objects and the Heliosphere, ISSI Sci Rep Ser, vol. 13, p 1Springer Science+Business Media, New York, doi:10.1007/978-1-4614-6384-9.

Quémerais, E., B. McClintock, G. Holsclaw, O. Katushkina, and V. Izmodenov (2014), Hydrogen atoms in the inner heliosphere: SWAN-SOHO and MASCS-MESSENGER observations, J. Geophys. Res. Space Physics, 119, 8017-8029, doi:10.1002/2014JA019761.

Ruciński, D., and M. Bzowski (1995), Modulation of interplanetary hydrogen density distribution during the solar cycle, Astron. Astrophys., 269, 248-263.

Snow, M., A. Reberac, E. Quémerais, J. Clarke, W. McClintock, and T. Woods (2013), A new catalog of ultraviolet stellar spectra for calibration, in Cross-Calibration of Far UV Spectra of Solar System Objects and the Heliosphere, edited by E. Quémerais, M. Snow, and R.-M. Bonnet, pp. 191-226, Springer, New York.

Stern, A., et al. (2008), ALICE: The ultraviolet imaging spectrograph aboard the New Horizons Pluto-Kuiper Belt Mission, Space Sci. Rev., 140, 155-187, doi:10.1007/s11214-008-9407-3.

Villanueva, G., M. Mumma, R. Novak, H. Käufl, P. Hartough, T. Encrenaz, A. Tokunaga, A. Khayat, and M. Smith (2015), Strong water isotopic anomalies in the Martian atmosphere: Probing current and ancient reservoirs, Science, 1-9, doi:10.1126/science.aaa3630.

Vincent, F. E., O. A. Katushkina, L. Ben-Jaffel, W. M. Harris, V. Izmodenov, E. Quémerais, D. Koutroumpa, and J. Clarke (2014), Observations of the interplanetary hydrogen during solar cycle 23 and 24 . What can we deduce about the local interstellar medium?, Astrophys. J. Lett. 788, L25.

Vincent, F., L. Ben-Jaffel, and W. Harris (2011), Updated analysis of the upwind Interplanetary Hydrogen velocity as observed by the Hubble Space Telescope during Solar Cycle 23, Astrophys. J., 738, 135, doi:10.1088/0004-637X/738/2/135. 